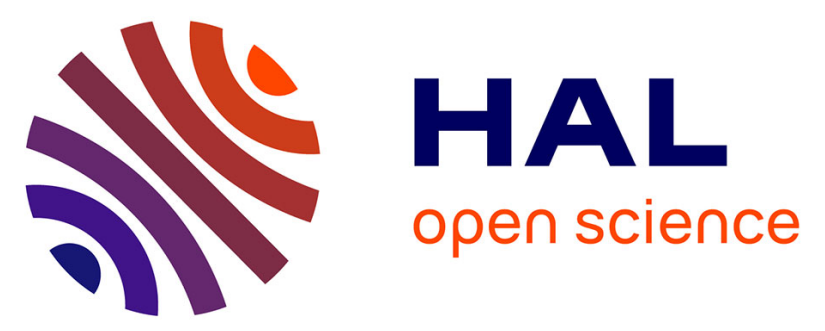

\title{
Highly Repeatable Dissolution Dynamic Nuclear Polarization for Heteronuclear NMR Metabolomics
} Aurélien Bornet, Mickael Maucourt, Catherine Deborde, Daniel Jacob, Jonas

Milani, Basile Vuichaud, Xiao Ji, Jean-Nicolas Dumez, Annick Moing, Geoffrey Bodenhausen, et al.

\section{To cite this version:}

Aurélien Bornet, Mickael Maucourt, Catherine Deborde, Daniel Jacob, Jonas Milani, et al.. Highly Repeatable Dissolution Dynamic Nuclear Polarization for Heteronuclear NMR Metabolomics. Analytical Chemistry, 2016, 88 (12), pp.6179-6183. 10.1021/acs.analchem.6b01094 . hal-01366193

\section{HAL Id: hal-01366193 https://hal.science/hal-01366193}

Submitted on 21 Jan 2021

HAL is a multi-disciplinary open access archive for the deposit and dissemination of scientific research documents, whether they are published or not. The documents may come from teaching and research institutions in France or abroad, or from public or private research centers.
L'archive ouverte pluridisciplinaire HAL, est destinée au dépôt et à la diffusion de documents scientifiques de niveau recherche, publiés ou non, émanant des établissements d'enseignement et de recherche français ou étrangers, des laboratoires publics ou privés. 


\title{
Highly repeatable dissolution dynamic nuclear polarization for heteronuclear NMR metabolomics
}

Aurélien Bornet ${ }^{1}$, Mickaël Maucourt²,3, Catherine Deborde ${ }^{2,4}$, Daniel Jacob²,4, Jonas Milani', Basile Vuichoud ${ }^{1}$, Xiao Ji¹, Jean-Nicolas Dumez ${ }^{5}$, Annick Moing ${ }^{2,4}$, Geoffrey Bodenhausen ${ }^{1,6,7,8}$, Sami Jannin $^{1, *}$, Patrick Giraudeau ${ }^{9,10, *}$

1. Institut des Sciences et Ingénierie Chimiques, Ecole Polytechnique Fédérale de Lausanne (EPFL), 1015 Lausanne, Switzerland. 2. Plateforme Métabolome Bordeaux - MetaboHUB, Centre de Génomique Fonctionnelle Bordeaux, IBVM, Centre INRA Bordeaux, 33140 Villenave d'Ornon, France. 3. Université de Bordeaux, UMR 1332 Biologie du Fruit et Pathologie, Centre INRA Bordeaux, 33140 Villenave d'Ornon, France. 4. INRA, UMR 1332 Biologie du Fruit et Pathologie, Centre INRA Bordeaux, 33140 Villenave d'Ornon, France. 5. Institut de Chimie des Substances Naturelles, CNRS UPR 2301, Univ. Paris-Sud, Université Paris-Saclay, 91190 Gif-sur-Yvette, France. 6. Département de Chimie, Ecole Normale Supérieure (ENS)-Paris Sciences Lettres (PSL) Research University, 75005 Paris, France. 7. Laboratoire de Biomolécules (LBM), Université Pierre et Marie Curie (UPMC) - Paris o6, Sorbonne Universités, 75005 Paris, France. 8. Laboratoire de Biomolécules (LBM), Unité Mixte de Recherche (UMR) 7203 Centre National de la Recherche Scientifique (CNRS), 75005 Paris, France. 9. Université de Nantes, CNRS, CEISAM UMR 6230, 44322 Nantes Cedex 03, France. 1o. Institut Universitaire de France, 75005 Paris, France.

\begin{abstract}
At natural ${ }^{13} \mathrm{C}$ abundance, metabolomics based on heteronuclear NMR is limited by sensitivity. We have recently demonstrated how hyperpolarization by dissolution dynamic nuclear polarization (D-DNP) assisted by cross-polarization (CP) provides a reliable way of enhancing the sensitivity of heteronuclear NMR in dilute mixtures of metabolites. In this Technical Note, we evaluate the precision of this experimental approach, a critical point for applications to metabolomics. The higher the repeatability, the greater the likelihood that one can detect small biologically relevant differences between samples. The average repeatability of our state-of-the-art D-DNP NMR equipment for samples of metabolomic relevance (20 mg dry weight tomato extracts) is $3.6 \%$ for signals above the limit of quantification (LOQ), and $6.4 \%$ when all the signals above the limit of detection (LOD) are taken into account. This first report on the repeatability of D-DNP highlights the compatibility of the technique with the requirements of metabolomics, and confirms its potential as an analytical tool for such applications.
\end{abstract}

Metabolomics is increasingly used to examine metabolic changes due to environmental or genetic modulations in different fields such as health, food and environmental sciences'. Advanced analytical methods are employed to study a broad array of biological samples, such as plant, animal or human extracts, biofluids, biopsies, etc. NMR and MS are the most commonly used analytical methods in the field of metabolomics as tens to hundreds of metabolites can be identified in a single experiment ${ }^{2}$. MS can detect thousands of metabolites with high sensitivity, but the quantitation by MS is less reproducible than by NMR, and the identification of biomarkers can be ambiguous ${ }^{3}$. While less sensitive, NMR is very reproducible for quantitation and enables the unambiguous identification of molecular structures through multi-nuclear and multi-dimensional methods. NMR and MS are therefore fully complementary, and numerous studies have shown that their combination can be beneficial for a better understanding of metabolic pathways ${ }^{4-6}$.

With current state-of-the-art proton NMR (high-field magnets, cryogenic probes, micro-coils...), one can detect biological samples with concentrations as low as $1 \mu \mathrm{M}^{7}$. Unfortunately, proton NMR suffers from extensive overlaps, particularly for biological samples, which is detrimental to the identification and quantitation of metabolite signals.

Heteronuclear NMR benefits from much larger ranges of chemical shifts of low-gamma nuclear spins such as ${ }^{13} \mathrm{C}$ and ${ }^{15} \mathrm{~N}^{8}$. However, heteronuclear NMR methods at natural abundance are not sensitive enough for detecting metabolites in complex mixtures, and they are rarely used for metabolomics. The vast majority of routine NMR metabolomics measurements rely on standardized ${ }^{~} \mathrm{H}$ NMR proto$\operatorname{cols}^{2,9,10}$. However NMR metabolomics would greatly benefit from heteronuclear spectroscopy if the sensitivity could be significantly boosted.

We have recently demonstrated how hyperpolarization by dissolution dynamic nuclear polarization (D-DNP) assisted by cross-polarization $(\mathrm{CP})^{11}$ provides a reliable way of enhancing the ${ }^{13} \mathrm{C}$ NMR sensitivity in dilute mixtures of metabolites ${ }^{12}$. The D-DNP approach was initially proposed by Golman et al. ${ }^{13}$ and further optimized as described in Fig. 1. The sample to be analyzed is dissolved in a glassforming solvent mixture doped with free radicals, frozen at 
temperatures below $4 \mathrm{~K}$, and hyperpolarized by microwave irradiation at a frequency close to the Larmor frequency of the unpaired electron. After rapid melting and transport of the hyperpolarized sample to a solution-state NMR spectrometer, enhancements of the polarization by factors up to $5 \mathrm{X10}^{4}$ can be achieved, providing a much-needed boost in sensitivity to solution-state NMR experiments.

In our D-DNP experiments, ca. 10 s are needed to transfer the sample from the polarizer to the NMR spectrometer. This can lead to significant losses of hyperpolarization through $T_{1}$ relaxation. Therefore, D-DNP works best for slow-relaxing nuclei such as ${ }^{13} \mathrm{C}$ or ${ }^{15} \mathrm{~N}$. D-DNP offers interesting perspectives for metabolomics based on heteronuclear NMR, but the question of its analytical performance has not yet been addressed. Most metabolomics studies rely on a comparison of the relative amplitudes of metabolite signals of samples from different groups. The precision is therefore critical and the repeatability must be as high as possible to reveal small differences. Although trueness (i.e., the closeness of the agreement between the measured value and the true concentration) is not essential here, it could be improved by proper calibration or standard addition procedures ${ }^{14}$.

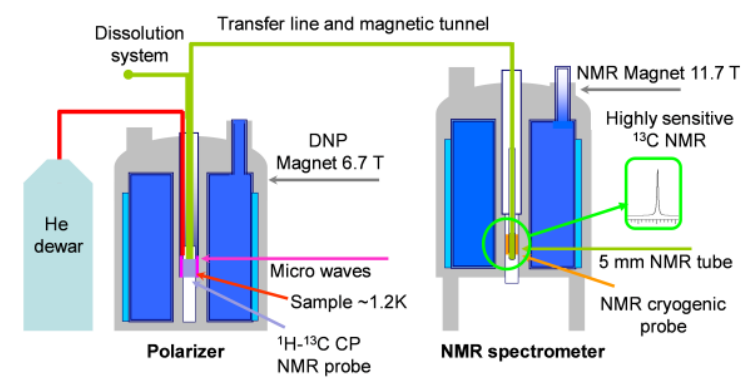

Figure 1. Equipment for dissolution-DNP NMR experiments.

Here, we evaluate for the first time the repeatability of CP-assisted D-DNP NMR experiments in the context of ${ }^{13} \mathrm{C}$ metabolomics. The instrumental repeatability is first assessed on concentrated ${ }^{13} \mathrm{C}$-labeled metabolites, then for plant extracts of metabolomic relevance with natural abundance ${ }^{13} \mathrm{C}$. Our observations demonstrate that the repeatability of our experimental setting matches the requirements of metabolomics. Future improvements are suggested that should enhance the performance even further.

\section{EXPERIMENTAL}

\section{Standard mixture of metabolites}

To evaluate the instrumental repeatability, a standard solution containing five $50 \mathrm{mM}$ metabolites was prepared: $\left[1^{-13} \mathrm{C}\right]$ L-alanine, $\left[1^{-{ }^{13}} \mathrm{C}\right]$ sodium acetate, $\left[{ }^{13} \mathrm{C}\right]$ sodium formate, and $\left[{ }^{13} \mathrm{C}\right]$ urea, dissolved in $1 \mathrm{~mL}$ of a $\mathrm{H}_{2} \mathrm{O}: \mathrm{D}_{2} \mathrm{O}$ :Glycerol-d $\mathrm{d}_{8}(1: 4: 5)$ mixture doped with $50 \mathrm{mM}$ TEMPOL (4-hydroxy-2,2,6,6-tetramethylpiperidin-1-oxyl). For each dissolution experiment, $80 \mu \mathrm{L}$ of the standard solution were transferred to the sample holder (the same being used for all experiments). All standard solutions were contained within the active volume of the radio-frequency $(r f)$ coil of the polarizer.

\section{Preparation of plant extracts}

Tomatoes (Solanum lycopersicum, L. Moneymaker variety) plants were grown following the protocol described previously ${ }^{6,12}$, and green fruits ( 28 days post anthesis) were harvested. For each fruit, a quarter of the fruit pericarp from the equatorial zone was immediately frozen in liquid nitrogen. The frozen sample was ground in liquid nitrogen and stored in $\mathrm{a}-80{ }^{\circ} \mathrm{C}$ freezer until freeze-drying. Ten extracts were prepared, each from $20 \mathrm{mg}$ lyophilized powder sample. Polar metabolites were extracted with a series of four ethanol-water solutions at $80^{\circ} \mathrm{C}$ following the protocol described in Ref. ${ }^{12}$. Each of the ten supernatant solutions was dried under vacuum, then solubilized in $500 \mu \mathrm{L}$ MilliQ water. The ten supernatant solutions were pooled, freezedried, and dissolved in $2 \mathrm{~mL}$ of a $\mathrm{H}_{2} \mathrm{O}: \mathrm{D}_{2} \mathrm{O}:$ Glycerol- $\mathrm{d}_{8}$ (1:4:5) mixture doped with 50 mM TEMPOL. Ethylene diamine tetraacetate disodium salt (EDTA $\mathrm{Na}_{2}, 2 \mathrm{mM}$ final concentration) was added to attenuate paramagnetic effects on the ${ }^{13} \mathrm{C}$ NMR detection of carboxylic metabolites such as citrate, malate, fumarate or various amino-acids. From this stock solution, eight aliquots of $200 \mu \mathrm{L}$ each were transferred to the sample holder, taking the same precautions as for the standard samples.

For conventional NMR analysis, an additional $20 \mathrm{mg}$ extract was solubilized in $700 \mu \mathrm{L} \mathrm{D}_{2} \mathrm{O}$ with $2 \mathrm{mM}$ EDTA $\mathrm{Na}_{2}$ and transferred to a $5 \mathrm{~mm}$ NMR tube.

\section{Dynamic Nuclear Polarization}

DNP was performed at $1.2 \mathrm{~K}$ and $6.7 \mathrm{~T}$ in a home-built polarizer by polarizing protons via microwave irradiation at $f_{\mu \mathrm{w}}=188.3 \mathrm{GHz}$ and $P_{\mu \mathrm{w}}=87.5 \mathrm{~mW}$ with frequency modulation over a range of $\Delta f_{\mu \mathrm{w}}=50 \mathrm{MHz}$ at a rate $f_{\text {mod }}=10 \mathrm{kHz}$. The proton polarization was transferred to ${ }^{13} \mathrm{C}$ by 7 consecutive cross-polarization (CP) contacts at intervals of $4 \mathrm{~min}$, with $10 \mathrm{~W}$ applied to ${ }^{1} \mathrm{H}$ and $120 \mathrm{~W}$ to ${ }^{13} \mathrm{C}$ (radio frequency amplitudes of $20 \mathrm{kHz}$ on both channels). After the $7^{\text {th }} \mathrm{CP}$ contact (i.e. after $28 \mathrm{~min}$ ), the samples were dissolved with $5 \mathrm{~mL}$ of $\mathrm{D}_{2} \mathrm{O}$ (preheated to $\mathrm{T}=450 \mathrm{~K}$ at $\mathrm{P}=1.0 \mathrm{MPa}$ ), taking care to dissolve always in the same magnetic field and at the same temperature, at the same height above the helium bath. The samples were then transferred with helium gas at o.6 MPa to a $500 \mathrm{MHz}$ NMR spectrometer through a $1.5 \mathrm{~mm}$ inner diameter PTFE tube in a o.8 T magnetic tunnel ${ }^{15}$, and injected into a $5 \mathrm{~mm}$ NMR tube. The complete dissolution, transfer and injection took $9.2 \mathrm{~s}$.

\section{NMR Spectroscopy}

All D-DNP enhanced NMR experiments were recorded at $298 \mathrm{~K}$ on a $500 \mathrm{MHz}$ Bruker Avance spectrometer equipped with an inverse cryogenic probe with triple axis gradients. The ${ }^{13} \mathrm{C}$ spectra of the hyperpolarized standard metabolite mixture were recorded in a single scan after excitation with a $5^{\circ}$ pulse with a $2.1 \mathrm{~s}$ acquisition time without ${ }^{1} \mathrm{H}$ decoupling, then processed with $3 \mathrm{~Hz}$ Lorentzian linebroadening and zero-filled to $128 \mathrm{~K}$ data points. The ${ }^{13} \mathrm{C}$ spectra of the hyperpolarized plant extracts were recorded 
after a $90^{\circ}$ pulse in a single scan with a $4.7 \mathrm{~s}$ acquisition time and Waltz-16 ${ }^{1} \mathrm{H}$ decoupling during acquisition, then processed with a $1 \mathrm{~Hz}$ line-broadening and zero-filled to 128 K data points.

The conventional ${ }^{13} \mathrm{C}$ spectrum of the plant extract is the same as the reference spectrum shown in Ref. ${ }^{12}$. It was prepared using a conventional sample preparation (i.e. dissolved in $700 \mu \mathrm{L} \mathrm{D}_{2} \mathrm{O}$ ) as previously described ${ }^{12}$. The NMR acquisition was performed on a $700 \mathrm{MHz}$ Bruker Avance III HD equipped with a cryogenic probe for inverse detection with z-gradients. The spectrum was recorded in $11 \mathrm{~h} 43$ min (1024 scans) with an inverse-gated decoupling pulse sequence, with carefully calibrated $90^{\circ}$ pulses, a 40 s recovery delay and a $1.2 \mathrm{~s}$ acquisition time. Waltz-16 ${ }^{1} \mathrm{H}$ decoupling was applied during the acquisition, with $5 \mathrm{~Hz}$ linebroadening, and zero-filling to $128 \mathrm{k}$ data points. After Fourier transform, the baseline was automatically corrected with a polynomial of degree 3 .

\section{Data processing}

For experiments on standard samples, the peaks were integrated in Topspin 3.2. The spectra of extracts were processed in NMRProcFlow (http://www.nmrprocflow.org) where the peaks were aligned before integration. For each experiment, the ${ }^{13} \mathrm{C}$ peak integrals were normalized by dividing each integral by the sum of the integrals of interest. The coefficients of variations were calculated by dividing the standard deviation on successive experiments by the mean value.

\section{RESULTS AND DISCUSSION}

\section{Instrumental repeatability}

We first evaluated the instrumental repeatability of the experimental setting described in Fig. 1, under conditions where the signal-to-noise ratio (SNR) is sufficiently high to ensure that the repeatability of the integration is not affected by noise. As described above, this evaluation was performed on a standard mixture of ${ }^{13} \mathrm{C}$ labeled metabolites. The ${ }^{13} \mathrm{C}$ spectrum (Fig. 2) was detected with a single $5^{\circ}$ pulse after $\mathrm{CP}$-assisted hyperpolarization and dissolution. The SNR was between 1500 and 4000 for the 5 peaks, corresponding to signal enhancements $\varepsilon_{\text {DNP }}$ between 22000 and 41000 (calculated with respect to the thermal equilibrium signal at $11.4 \mathrm{~T}$ and $300 \mathrm{~K}$, measured over night with 128 scans and a recycle delay of $300 \mathrm{~s}$ ).

Under such conditions of high SNR, the repeatability of the NMR signals mostly reflects the stability of the instrumentation. Table 1 summarizes this variability through the coefficients of variation (CV) of the NMR peak areas measured from 8 successive experiments. The absolute variations are between 20 and 30\%, which is not surprising given the sources of variability involved in the experimental setting (i.e., polarization efficiency, sample dissolution and transfer, and sample homogeneity after dissolution). However, in the context of metabolomics, the relevant quantity is generally the normalized NMR signal, i.e., the area of an individual peak divided by the sum of all peak areas in the spectrum ${ }^{14}$. Table 1 shows that after such a normalization, the repeatability lies between $1.4 \%$ and $4.3 \%$. These values are much smaller than the biological variability, which is generally at least $10-20 \%$ in metabolomics studies. This result demonstrates that after normalization, the repeatability of our D-DNP experiments is sufficient to meet the precision requirements of metabolomics. In future developments, we could consider the introduction of an internal reference compound of known concentration as an alternative to data normalization.

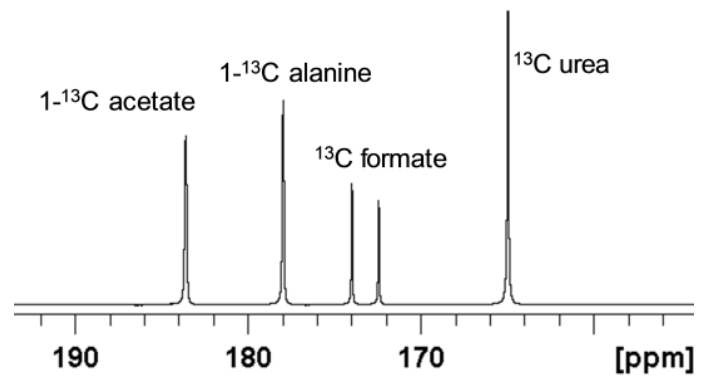

Figure 2. Single-scan ${ }^{13} \mathrm{C}$ NMR spectrum of a standard mixture containing four ${ }^{13} \mathrm{C}$ labeled metabolites, recorded in a single scan with a $5^{\circ}$ pulse after D-DNP combined with CP. The extract was dissolved in a $200 \mu \mathrm{L}$ mixture of $\mathrm{H}_{2} \mathrm{O} / \mathrm{D}_{2} \mathrm{O} /$ glycerol$\mathrm{d}_{8}(1: 4: 5)$ doped with $50 \mathrm{mM}$ TEMPOL, then polarized for 28 min at $1.2 \mathrm{~K}$ and $6.7 \mathrm{~T}$, and finally dissolved with $5 \mathrm{~mL}$ of hot $\mathrm{D}_{2} \mathrm{O}$ and transferred to a $500 \mathrm{MHz}$ spectrometer equipped with a cryogenic probe. $\mathrm{No}^{1} \mathrm{H}$ decoupling was applied here.

Table 1. Instrumental repeatability of our D-DNP NMR experiments for eight successive ${ }^{13} \mathrm{C}$ spectra of a mixture of four ${ }^{13} \mathrm{C}$ labeled metabolites (as in Fig. 2). The coefficients of variation (CV) are determined both for the integrals of individual peaks, and for relative integrals normalized to the total integral.

\begin{tabular}{cccccc} 
& Alanine & Acetate & \multicolumn{2}{c}{ Formate } & Urea \\
\hline$\delta(\mathrm{ppm})$ & 183.7 & 178.0 & 174.1 & 172.5 & 165.0 \\
$\mathrm{CV} \quad$ (in- & & & & & \\
$\begin{array}{c}\text { dividual } \\
\text { peaks) }\end{array}$ & $26.6 \%$ & $24.7 \%$ & $30.1 \%$ & $29.3 \%$ & $28.8 \%$ \\
$\begin{array}{c}\mathrm{CV} \\
\text { (norm. }\end{array}$ & $1.4 \%$ & $4.3 \%$ & $3.0 \%$ & $2.7 \%$ & $2.0 \%$ \\
peaks) & & & & & \\
\hline
\end{tabular}

The actual sources of variability in the ${ }^{13} \mathrm{C}$ NMR signal after D-DNP were systematically evaluated (see Supplement). Sample preparation and spectrometer stability lead to minute variations of about $1 \%$, while the manual dissolution process leads to $22 \%$ variations. These variations will in principle be reduced dramatically by fully automating the dissolution process. Uncertainties in the transfer time, here typically $100 \mathrm{~ms}$, do not lead to significant variation $\sin { }^{13} \mathrm{C}$ intensities between analytes with different T1's, here less than $0.5 \%$. 


\section{Repeatability under conditions typical for metabolomics}

In a metabolomics perspective, the repeatability should be evaluated under conditions that are realistic for metabolomics, i.e., on biological samples at natural ${ }^{13} \mathrm{C}$ abundance. To do so, we prepared 8 identical extracts from tomato fruit pericarp. To remove sources of biological variability, all extracts were pooled and dissolved in a DNP mixture, then aliquoted as described in the experimental part. The single-scan ${ }^{13} \mathrm{C}$ spectrum of one of these hyperpolarized extracts is shown in Fig. 3 and compared to a conventional signal-averaged spectrum of a similar but seven-fold more concentrated extract (same mass of extract but dissolved in $700 \mu \mathrm{L} \mathrm{D} 2 \mathrm{O}$ for conventional NMR analysis). The spectra in Fig. 3 focuses on the quaternary ${ }^{13} \mathrm{C}$ region, where the sensitivity gain is the most remarkable and where the polarization is best preserved because of favorably long $\left.T_{1}{ }^{13} \mathrm{C}\right)$. The limitation to quaternary ${ }^{13} \mathrm{C}$ nuclei is not necessarily crippling for metabolomics applications, since many metabolites possess at least one quaternary carbon, but we shall extend this study to all carbons in the near future, provided we can reduce the transfer time from ca. 10 to $c a$. $1 \mathrm{~s}$ as demonstrated by C. Hilty and co-workers ${ }^{16}$. With our current instrumentation, we can only obtain large sensitivity gains in the quaternary ${ }^{13} \mathrm{C}$ region. Still, the single-scan hyperpolarized ${ }^{13} \mathrm{C}$ spectrum shows many more peaks than the thermal spectrum, even though the latter was recorded in $c a .12 \mathrm{~h}$. Note that the peaks observed on the conventional spectrum do not necessarily appear at the same chemical shifts as in the hyperpolarized spectrum, since the $\mathrm{pH}$ and solvent are different.

In NMR metabolomics, a standard data processing method consists in dividing the spectra into user-defined regions called 'buckets', the integrals of which serve as variables for statistical analysis ${ }^{17}$. The quality of integration can be further improved through peak alignment-based procedures ${ }^{18}$. The hyperpolarized ${ }^{13} \mathrm{C}$ spectra of our extracts (Fig. 3a) were subjected to this approach, leading to 26 buckets where signals above the limit of detection (LOD, defined by SNR > 3) ${ }^{19}$ could be observed. These buckets were normalized by the sum of all buckets, and their repeatability was evaluated through 8 successive dissolutions. The coefficients of variation are shown in Fig. 4, together with the SNRs of the corresponding buckets. Here, the repeatability appears to be dominated by the SNR, as in NMR experiments without hyperpolarization. When all buckets above the LOD are considered, the average repeatability is $6.4 \%$ and the CV is inversely proportional to the SNR. But if one considers only the 16 buckets above the limit of quantification (LOQ, defined by SNR > $10)^{19}$, the average repeatability is improved to $3.6 \%$ while all CVs are below $10 \%$. Thus, the precision of D-DNP NMR experiments appears to be sufficient for metabolomics as long as the SNR remains above the LOQ.

\section{CONCLUSIONS}

This note shows that the precision of CP-assisted D-DNP experiments is sufficient to satisfy the requirements of NMR metabolomics, provided the integrated signals are above the LOQ (i.e., provided their SNR > 10). The remaining variations on relative peak areas (3.6\% on average) may originate from different sources. The main source of metabolite-dependent variations is the duration of the dissolution and transfer steps, since their non-repeatability leads to variable relaxation losses during the transfer step. Automating the dissolution process may reduce these variations even further.

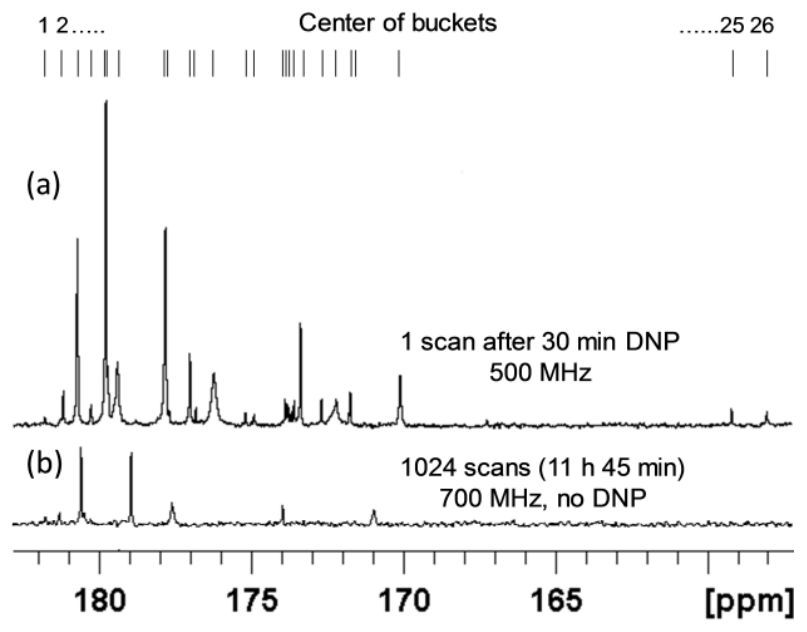

Figure 3. Quaternary region of ${ }^{13} \mathrm{C}$ NMR spectra of green tomato fruit pericarp extracts. (a) Single-scan ${ }^{13} \mathrm{C}$ NMR spectrum of a $20 \mathrm{mg}$ extract (prepared from $20 \mathrm{mg}$ lyophilized ground tissue) recorded with a single $90^{\circ}$ pulse after D-DNP boosted by $\mathrm{CP}$. The extract was first dissolved in $200 \mu \mathrm{L}$ of a mixture of $\mathrm{H}_{2} \mathrm{O} / \mathrm{D}_{2} \mathrm{O} /$ glycerol- $_{8}$ (1:4:5) doped with $50 \mathrm{mM}$ TEMPOL, then polarized for $28 \mathrm{~min}$ at $1.2 \mathrm{~K}$ and $6.7 \mathrm{~T}$, and finally dissolved with $5 \mathrm{~mL}$ of hot $\mathrm{D}_{2} \mathrm{O}$ and transferred to a $500 \mathrm{MHz}$ spectrometer equipped with a cryogenic probe. The lines above the spectrum indicate the center of the 26 spectral regions ('bucket') that were used for signal integration. (b) Conventional spectrum -obtained without hyperpolarization- of an identical extract dissolved in $700 \mu \mathrm{L} \mathrm{D}_{2} \mathrm{O}$, recorded with 1024 scans (11 h $45 \mathrm{~min}$ ) at $700 \mathrm{MHz}$ using a cryogenic probe. No attempt was made to assign the peaks, since the two spectra were recorded under different $\mathrm{pH}$ and temperature conditions.

Applications of D-DNP technology to high-throughput metabolomics will require reducing the transfer and injection times ${ }^{16}$ so as to maximize the number of ${ }^{13} \mathrm{C}$ signals above the LOQ. Reducing this time from 10 to 1 s would not dramatically improve the sensitivity of quaternary carbon signals (one would merely expect a gain of $16 \%$ if $T_{1}\left({ }^{13} \mathrm{C}\right)=$ $60 \mathrm{~s})$, but would result in a 5 -fold gain for protonated carbons, assuming $T_{1}\left({ }^{13} \mathrm{C}\right)=5 \mathrm{~s}$. Other technological improvements will be implemented to improve the repeatability, such as a fully automated dissolution procedure and a better control of the microwave irradiation. These improvements would significantly broaden the perspectives of applications of D-DNP to metabolomics. Moreover, the coupling of D-DNP with more sophisticated NMR methods such as single-scan $2 \mathrm{D}$ NMR will increase the amount of information that can be obtained from D-DNP ${ }^{12,20}$. This approach will significantly reduce peak overlap while provid- 
ing useful assignment information. Peak assignment strategies will be investigated in the future, with careful control of the $\mathrm{pH}$ conditions and/or on the spiking of the biological samples with commercial standards. Finally, potential interactions between the polarizing radical and the metabolites will be evaluated, and possibly removed if necessary by the addition of chelating agents (such as EDTA) or by using hybrid polarizing solids (HYPSO) ${ }^{21}$. Meanwhile, we hope that this first report on the precision of D-DNP will help highlighting its potential as a new analytical tool for the analysis of complex metabolic mixtures.

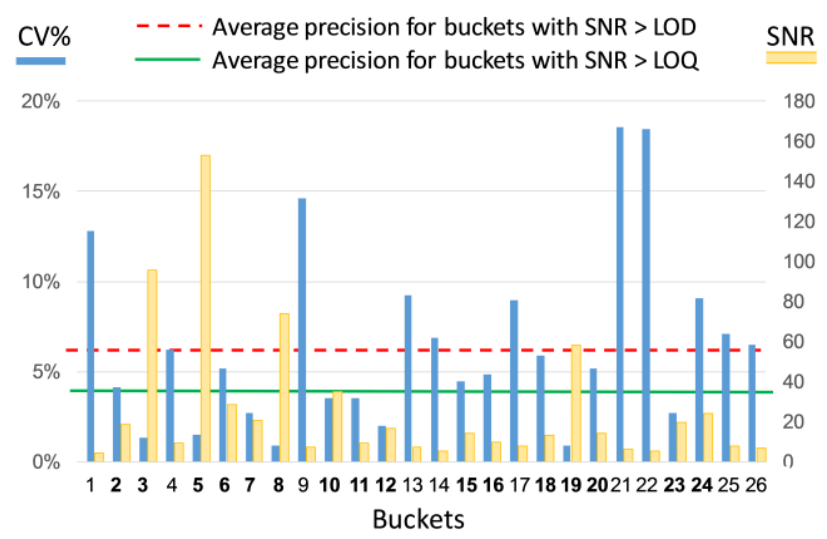

Figure 4. Repeatability and sensitivity of D-DNP ${ }^{13} \mathrm{C}$ NMR for metabolomics. The blue bars correspond to the coefficients of variation (CVs) of the individual bucket areas normalized to the sum of all areas, determined for 8 successive experiments performed on identical samples. The average precision, expressed in terms of repeatability, is $6.4 \%$ for all buckets containing signals above the limit of detection (LOD), and $3.6 \%$ when only the 16 buckets with signals above the limit of quantification (LOQ) are taken into account. The dashed orange bars correspond to the SNR (average value determined for the 8 spectra) of the corresponding buckets, showing that the repeatability is proportional to the inverse of the SNR. The buckets are numbered from 1 to 26 , indicated by lines on top of Figure 3 (left to right). Those in bold indicate buckets containing signal above the LOQ.

\section{AUTHOR INFORMATION}

\section{Corresponding Author}

* To whom correspondence should be addressed: patrick.giraudeau@univ-nantes.fr / sami.jannin@epfl.ch

\section{Author Contributions}

All authors have given approval to the final version of the manuscript.

\section{ACKNOWLEDGMENT}

PG acknowledges funding from the CORSAIRE metabolomics platform (Biogenouest network). SJ, AB and GB thank Anto Barisic and Dr Pascal Miéville at EPFL for valuable assistance, acknowledge funding from the Swiss National Science Foundation (SNSF), the Ecole Polytechnique Fédérale de Lausanne (EPFL), the Swiss Commission for Technology and Innovation (CTI), the CNRS, and the European Research Council (ERC contract No. 339754 "Dilute para-water"), and support by Bruker BioSpin. MM, CD and AM thank MetaboHUB ANR-11INBS-o1o and Eranet-EraSysBio+ 'FRuit Integrative Modelling' project.

\section{REFERENCES}

(1) Griffiths, W. J. Metabolomics, Metabonomics and Metabolite Profiling; Cambridge RSC Publishing, 2008.

(2) Bouatra, S.; Aziat, F.; Mandal, R.; Guo, A. C.; Wilson, M. R.; Knox, C.; Bjorndahl, T. C.; Krishnamurthy, R.; Saleem, F.; Liu, P.; Dame, Z. T.; Poelzer, J.; Huynh, J.; Yallou, F. S.; Psychogios, N.; Dong, E.; Bogumil, R.; Roehring, C.; Wishart, D. S. PLoS ONE 2013, 8, e73076.

(3) Pan, Z.; Raftery, D. Anal. Bioanal. Chem. 2oo6, 387, 525-527.

(4) Lanza, I. R.; Zhang, S.; Ward, L. E.; Karakelides, H.; Raftery, D.; Nair, K. S. PLoS ONE 2010, 5, e10538.

(5) Dai, H.; Xiao, C.; Liu, H.; Tang, H. J. Proteome Res. 2010, 9, 146o1475.

(6) Bénard, C.; Bernillon, S.; Biais, B.; Osorio, S.; Maucourt, M.; Ballias, P.; Deborde, C.; Colombié, S.; Cabasson, C.; Jacob, D.; Vercambre, G.; Gautier, H.; Rolin, D.; Génard, M.; Fernie, A. R.; Gibon, Y.; Moing, A. J. Exp. Bot. 2015, 66, 3391-3404.

(7) Nagana Gowda, G. A.; Raftery, D. J. Magn. Reson. 2015, 26o, 144160.

(8) Clendinen, C. S.; Lee-McMullen, B.; Williams, C. M.; Stupp, G. S.; Vandenborne, K.; Hahn, D. A.; Walter, G. A.; Edison, A. S. Anal. Chem. 2014, 86, 9242-9250.

(9) Beckonert, O.; Keun, H. C.; Ebbels, T. M. D.; Bundy, J.; Holmes, E.; Lindon, J. C.; Nicholson, J. K. Nat. Protocols 2007, 2, 2692-2703.

(10) Allwood, J. W.; de Vos, C. H. R.; Moing, A.; Deborde, C.; Erban, A.; Kopka, J.; Goodacre, R.; Hall, R. Methods Enzymol. 2011, 500, 299336.

(11) Bornet, A.; Melzi, R.; Perez Linde, A. J.; Hautle, P.; van den Brandt, B.; Jannin, S.; Bodenhausen, G. J. Chem. Phys. Lett. 2013, 4, 111-114.

(12) Dumez, J.-N.; Milani, J.; Vuichoud, B.; Bornet, A.; Lalande-Martin,

J.; Tea, I.; Yon, M.; Maucourt, M.; Deborde, C.; Moing, A.; Frydman, L.; Bodenhausen, G.; Jannin, S.; Giraudeau, P. Analyst 2015, 140, 58605863.

(13) Ardenkjaer-Larsen, J. H.; Fridlund, B.; Gram, A.; Hansson, G.; Hansson, L.; Lerche, M. H.; Servin, R.; Thaning, M.; Golman, K. Proc. Natl. Acad. Sci. U. S. A. 2003, 100, 10158-10163.

(14) Giraudeau, P.; Tea, I.; Remaud, G. S.; Akoka, S. J. Pharm. Biomed. Anal. 2014, 93, 3-16.

(15) Milani, J.; Vuichoud, B.; Bornet, A.; Miéville, P.; Mottier, R.; Jannin, S.; Bodenhausen, G. Rev. Sci. Instrum. 2015, 86, 024101.

(16) Chen, H.-Y.; Hilty, C. ChemPhysChem 2015, 16, 2646-2652.

(17) Giraudeau, P.; Tea, I.; Remaud, G. S.; Akoka, S. Journal of pharmaceutical and biomedical analysis 2014, 93, 3-16.

(18) Jacob, D.; Deborde, C.; Moing, A. Anal. Bioanal. Chem 2013, 405, 5049-5061.

(19) ICH-Q2 (R1).

http://www.ich.org/fileadmin/Public Web Site/ICH Products/Guideli nes/Quality $/ Q_{2} R_{1} /$ Step $_{4} / Q_{2} \quad R_{1} \quad$ Guideline.pdf 1995.

(20) Giraudeau, P.; Shrot, Y.; Frydman, L. J. Am. Chem. Soc. 2009, 131, 13902-13903.

(21) Gajan, D.; Bornet, A.; Vuichoud, B.; Milani, J.; Melzi, R.; van Kalkeren, H. A.; Veyre, L.; Thieuleux, C.; Conley, M. P.; Grüning, W. R.; Schwarzwälder, M.; Lesage, A.; Copéret, C.; Bodenhausen, G.; Emsley, L.; Jannin, S. Proc. Natl. Acad. Sci. USA 2014, 111, 14693-14697. 


\section{Table of contents entry}

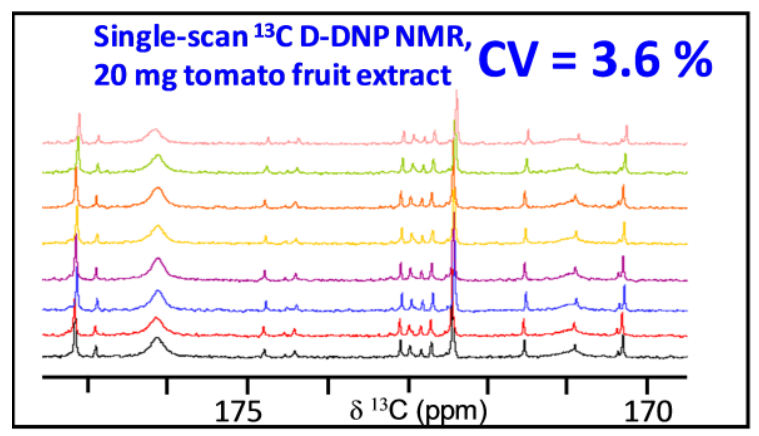




\title{
Supporting information for manuscript
}

\section{Highly repeatable dissolution dynamic nuclear polarization for heteronuclear NMR metabolomics}

\begin{abstract}
Aurélien Bornet ${ }^{1}$, Mickaël Maucourt ${ }^{2,3}$, Catherine Deborde ${ }^{2,4}$, Daniel Jacob ${ }^{2,4}$, Jonas Milani $^{1}$, Basile Vuichoud ${ }^{1}$, Xiao Ji ${ }^{1}$, Jean-Nicolas Dumez ${ }^{5}$, Annick Moing ${ }^{2,4}$, Geoffrey Bodenhausen $^{1,6,7,8}$, Sami Jannin ${ }^{1, *}$, Patrick Giraudeau ${ }^{9,10, *}$

1. Institut des Sciences et Ingénierie Chimiques, Ecole Polytechnique Fédérale de Lausanne (EPFL), 1015 Lausanne, Switzerland. 2. Plateforme Métabolome Bordeaux - MetaboHUB, Centre de Génomique Fonctionnelle Bordeaux, IBVM, Centre INRA Bordeaux, 33140 Villenave d'Ornon, France. 3. Université de Bordeaux, UMR 1332 Biologie du Fruit et Pathologie, Centre INRA Bordeaux, 33140 Villenave d'Ornon, France. 4. INRA, UMR 1332 Biologie du Fruit et Pathologie, Centre INRA Bordeaux, 33140 Villenave d'Ornon, France. 5. Institut de Chimie des Substances Naturelles, CNRS UPR 2301, Univ. Paris-Sud, Université ParisSaclay, 91190 Gif-sur-Yvette, France. 6. Département de Chimie, Ecole Normale Supérieure (ENS)-Paris Sciences Lettres (PSL) Research University, 75005 Paris, France. 7. Laboratoire de Biomolécules (LBM), Université Pierre et Marie Curie (UPMC) - Paris o6, Sorbonne Universités, 75005 Paris, France. 8. Laboratoire de Biomolécules (LBM), Unité Mixte de Recherche (UMR) 7203 Centre National de la Recherche Scientifique (CNRS), 75005 Paris, France. 9. Université de Nantes, CNRS, CEISAM UMR 6230, 44322 Nantes Cedex 03, France. 10. Institut Universitaire de France, 75005 Paris, France.
\end{abstract}

\section{Content:}

Additional discussion on the sources of irreproducibility in D-DNP experiments Page S-2

Additional tables and figures on the repeatability of the D-DNP experiment. Page S-4

Table S-1 Page S-4

Figure S-1. Page S-4

Figure S-2. Page S-5

Table S-2. Page S-5 


\section{Additional discussion on the sources of irreproducibility in D-DNP experiments}

The potential sources of variability in the ${ }^{13} \mathrm{C}$ NMR signal after D-DNP can be classified into two categories: those affecting all the analytes identically and those leading to analyte-dependent variations. These two categories are discussed below.

\section{1) Analyte-independent variations}

These variations explain the absolute peak area variations observed in Table 1, and may arise from different sources:

Sample amount variations is negligible: The mass of sample that is transferred to the DNP sample cup can slightly vary, due to imprecisions in measuring the sample volume. Here, the sample was systematically weighted before analysis, and the mass variations are reported in Table S-1. As can be seen from these variations ( $1.1 \%)$, the sample weighting is very reproducible and does not bring a significant contribution to the signal variability.

Spectrometer stability is good enough: Quantitative ${ }^{13} \mathrm{C}$ NMR experiments are now performed with a repeatability better than $1 \%$ on modern hardware (see for example E. Caytan et al., Talanta 2007, 71, 1016-1021), therefore we can reasonably assume that this is not significantly impacting our measurements.

We will neglect these two first sources of variability that are an order of magnitude smaller than the following ones.

Dissolution operator-dependent variations leads to significant variation in polarization ( $\mathrm{V}^{\mathrm{P}}$ operator). The dissolution process is currently a semi-automated step requiring intervention by an operator. The operator first manually lifts the sample insert by c.a. $10 \mathrm{~cm}$ over the liquid helium bath, subsequently manually inserts a dissolution device that physically couples to the sample holder. Finally, the operator pushes a button at the top of the dissolution device to trigger the automated dissolution sequence. Therefore, three critical factors can vary during which nuclear-spin lattice relaxation is active:

(1) the exact positioning of the sample insert, and therefore the magnetic field in which the sample starts to relax.

(2) the time to physically couple the dissolution device to the sample holder

(3) the time between (a) the manual coupling of the dissolution insert to the cold sample and (b) the manual triggering of dissolution by pushing a button on the dissolution insert. During this time, the sample warms up in the solid state and paramagnetic relaxation is enhanced.

These three operator dependent variations will necessarily affect the absolute signals detected in the liquid-state. These variations in dissolution steps may also impact the relative signal intensities (see next page).

Dissolution turbulent variations lead to significant variations in concentration $\left(\mathrm{V}_{\text {turbulent }}^{\mathrm{c}}\right)$. Additionally, even if full automation were made, the dissolution process is a very turbulent process, therefore one can still expect significant variations in final concentration after dissolution, transfer, and injection. Indeed, we have observed that part of the losses is simply due to the fact that only a variable fraction of the sample initially polarizes is actually injected in the detection NMR tube.

In order to quantify these sources of variation, we have measured the ${ }^{13} \mathrm{C}$ signals in the liquid state just after dissolution, which provide a global assessment of all contributions. In addition, we have measured the analyte concentrations by quantitative ${ }^{1} \mathrm{H}$ NMR after dissolution, at thermal equilibrium, which provides a specific measure of $\mathrm{V}^{\mathrm{c}}$ turbulent. Table $\mathrm{S}-1$ shows the total ${ }^{1} \mathrm{H}$ signal integrals, with an overall variation of $V_{\text {turbulent }}=22.1 \%$. This result highlights that there is a significant contribution of 
the automated part of the dissolution step in the signal variability. This variation is comparable to the absolute liquid-state ${ }^{13} \mathrm{C}$ signal variations. More interestingly, Figure S-2 shows that the ${ }^{13} \mathrm{C}$ signal variations are correlated to the relative concentrations detected by ${ }^{1} \mathrm{H}$ NMR, so that most of the variations come from the turbulent nature of the transfer process.

This contribution may be reduced by a better engineering of the fluid path, that would for example make the dissolution process more laminar and less turbulent. But here this contribution can be easily removed by normalizing the ${ }^{13} \mathrm{C}$ liquid state signal integrals by the quantitative ${ }^{1} \mathrm{H}$ liquid-state integrals at thermal equilibrium, which only leaves us with the operator dependent and DNP efficiency variability.

Solid-state DNP efficiency variations: DNP involves complex mechanisms and its efficiency is dependent on many factors such as the temperature, the microwave power and frequency stability, and the efficiency of the cross-polarization steps. The combined contribution of these effects to the signal variations observed in D-DNP ${ }^{13} \mathrm{C}$ spectra can in principle be evaluated by measuring the total ${ }^{13} \mathrm{C}$ signal integral in the solid-state right before dissolution. Table 1 shows the solid-state integrals measured just before the 8 successive dissolutions on the standard samples. These values show a significant variation (VDNP $=\mathbf{1 1 . 6 \%}$ ). These variations, however, are not correlated with the liquidstate ${ }^{13} \mathrm{C}$ signals detected after dissolution (Figure $\mathrm{S}-1$ ). This is probably because, in the current setup, the solid-state NMR measurements are not fully reliable because of tuning instabilities and approximate sample positioning, one cannot assign these variations to true differences of polarization.

\section{2) Analyte-dependent variations}

These variations will explain the relative peak area variations, and may arise from two main sources:

Transfer time between the two magnets does not lead to significant variations: each analyte relaxes with a different $T_{1}$, which can impact the relative ${ }^{13} \mathrm{C}$ NMR intensities after transfer. We therefore estimated the $\mathrm{T}_{1} \mathrm{~s}$ by measuring the ${ }^{13} \mathrm{C}$ signal decay for each targeted peak after dissolution with smallangle $\left(5^{\circ}\right)$ pulses at regular intervals $(5 \mathrm{~s})$. The resulting effective $\mathrm{T}_{1} \mathrm{~S}$ are shown in Table S-2. Based on these values, this Table also shows the relative intensity variations that would result from the current variations in transfer time by \pm 100 ms. The resulting variations are well below the relative errors observed in Table 1. This means that small variations in the transfer time are irrelevant for the relative variations observed in our study.

Dissolution step may lead to significant relative variations: As described above, when the sample warms up in the solid state, paramagnetic relaxation is enhanced in an analyte-dependent fashion. This effect is likely to explain the remaining relative signal variations.

\section{Conclusion:}

In conclusion, most of the absolute signal variations can be attributed to the operator dependent variations, which needs to be improved by a full automation of the dissolution process. This contribution can however be easily removed by normalizing the ${ }^{13} \mathrm{C}$ liquid state signal integrals by the quantitative ${ }^{1} \mathrm{H}$ liquid-state integrals at thermal equilibrium. The remaining relative signal variations can be explained by relaxation in the dissolution step. 
For the second part of the study (metabolic samples), these conclusions are still valid as long as the SNR is above the limit of quantification. When this condition is not fulfilled, additional variability sources arise from the irreproducibility of peak integration for low SNR values.

The tables and figures below provide additional information regarding the sources of irreproducibility of the D-DNP experiment. All the data were obtained from experiments performed on a standard mixture of four ${ }^{13} \mathrm{C}$ labeled metabolites (as described in the experimental part).

Table S-1. Instrumental repeatability of the D-DNP NMR experiment for eight successive ${ }^{13} \mathrm{C}$ spectra of a mixture of four ${ }^{13} \mathrm{C}$ labeled metabolites. Sample weight, solid-state ${ }^{13} \mathrm{C}$ integrals before dissolution, liquid-state ${ }^{13} \mathrm{C}$ integrals after dissolution and liquid-state ${ }^{1} \mathrm{H}$ integrals at thermal equilibrium after dissolution.

\begin{tabular}{|c|c|c|c|c|c|c|c|c|c|c|}
\hline \multirow{3}{*}{ Exp. } & \multirow{3}{*}{$\begin{array}{c}\text { Sample } \\
\text { weight } \\
\text { (mg) }\end{array}$} & \multirow{3}{*}{$\begin{array}{c}\text { Solid-state } \\
{ }^{13} \mathrm{C} \text { integral } \\
\text { before } \\
\text { dissolution } \\
\text { Chem. Shift }\end{array}$} & \multicolumn{5}{|c|}{ Liquid-state ${ }^{13} \mathrm{C}$ integrals } & \multicolumn{3}{|c|}{$\begin{array}{l}\text { Liquid-state }{ }^{1} \mathrm{H} \text { integrals } \\
\text { (thermal equilibrium) }\end{array}$} \\
\hline & & & Alanine & Acetate & For & tate & Urea & Alanine & Acetate & Formate \\
\hline & & & 183.7 & 178.0 & 174.1 & 172.5 & 165.0 & 1.37 & 1.81 & 8.34 \\
\hline 1 & 100.4 & 2.392 & 1.000 & 0.937 & 0.380 & 0.320 & 1.370 & 1.000 & 1.000 & 1.000 \\
\hline 2 & 99.2 & 1.998 & 0.876 & 0.866 & 0.312 & 0.271 & 1.129 & 1.024 & 1.006 & 0.957 \\
\hline 3 & 100.1 & 2.682 & 0.880 & 0.880 & 0.310 & 0.255 & 1.103 & 0.773 & 0.766 & 0.697 \\
\hline 4 & 101.5 & 2.430 & 0.914 & 0.933 & 0.320 & 0.270 & 1.177 & 0.909 & 0.896 & 0.829 \\
\hline 5 & 101.4 & 2.512 & 0.979 & 0.985 & 0.347 & 0.287 & 1.234 & 0.848 & 0.841 & 0.779 \\
\hline 6 & 99.5 & 2.182 & 1.555 & 1.496 & 0.582 & 0.492 & 2.098 & 1.394 & 1.371 & 1.260 \\
\hline 7 & 100.6 & 2.753 & 1.576 & 1.476 & 0.614 & 0.496 & 2.116 & 1.246 & 1.219 & 1.122 \\
\hline 8 & 98.3 & 2.066 & 1.370 & 1.353 & 0.512 & 0.434 & 1.801 & 1.390 & 1.355 & 1.274 \\
\hline $\begin{array}{c}\text { CV } \\
\text { (absolute } \\
\text { variation) }\end{array}$ & $1.1 \%$ & $11.6 \%$ & $26.6 \%$ & $24.7 \%$ & $30.1 \%$ & $29.3 \%$ & $28.8 \%$ & $22.5 \%$ & $22.0 \%$ & $21.9 \%$ \\
\hline
\end{tabular}

Chemical shifts are indicated in ppm. ${ }^{13} \mathrm{C}$ liquid-state integrals are absolute integrals (arbitrary scale). ${ }^{1} \mathrm{H}$ Liquid-state integrals are normalized to the first experiment for each analyte. The coefficients of variation (CV) are determined both for the integrals of individual peaks, and for relative integrals normalized to the total integral.

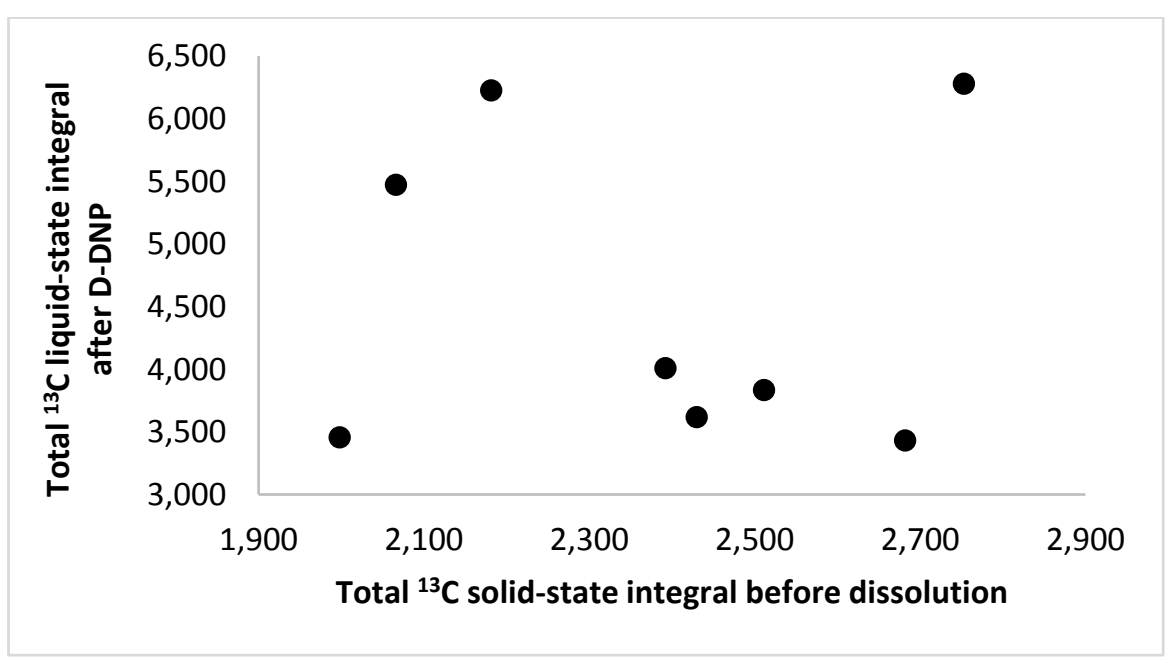

Figure S-1. Sum of the ${ }^{13} \mathrm{C}$ integrals of the four analytes (alanine, acetate, formate, urea) measured in the liquid state after D-DNP, as a function of the total ${ }^{13} \mathrm{C}$ integral in the solid-state right before the dissolution. No correlation is observed between these values. 

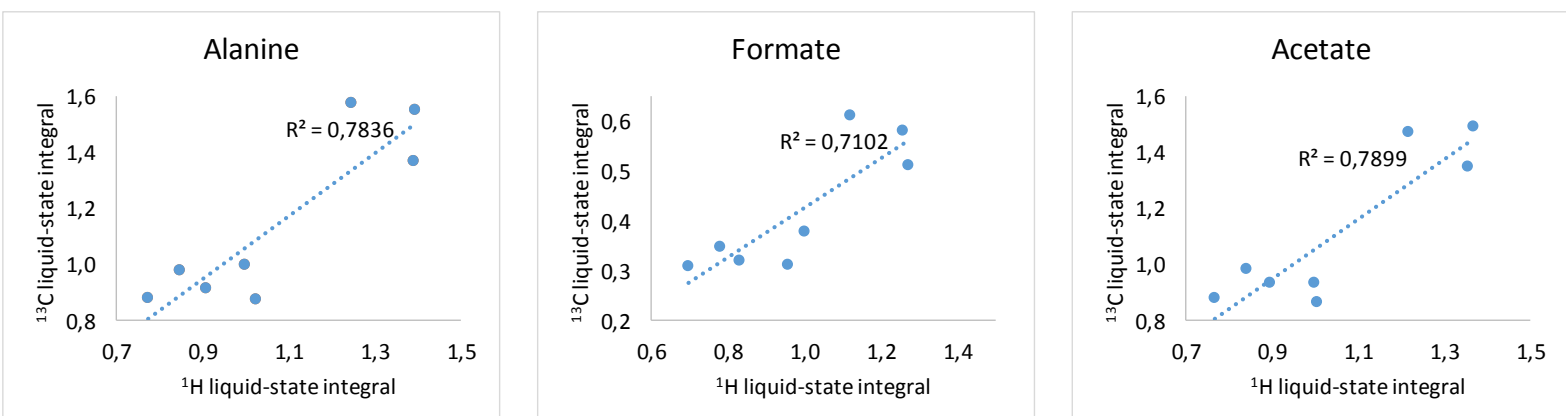

Figure S-2. ${ }^{13} \mathrm{C}$ liquid-state integrals measured after D-DNP versus ${ }^{1} \mathrm{H}$ integrals measured at thermal equilibrium from the same samples, for three ${ }^{13} \mathrm{C}$-labeled analytes of the standard mixture (alanine, formate and acetate, from left to right -urea did not show any ${ }^{1} \mathrm{H}$ signal since its protons were in fast exchange with water).

Table S-2. Effective ${ }^{13} \mathrm{C}$ longitudinal relaxation times for the carbons of interest in the ${ }^{13} \mathrm{C}$-labeled metabolite mixture, measured from a series of small-angle pulse experiments ( $5^{\circ}$ every $7.08 \mathrm{~s}$ ) after dissolution. Based on these values, the absolute and relative intensities that would result from a $\pm 1 \mathrm{~s}$ variation in the transfer time $\tau$ (relative to the current value of $9.2 \mathrm{~s}$ ) are calculated, as well as the corresponding variation in $\%$.

\begin{tabular}{|c|c|c|c|c|c|c|}
\hline & & Alanine & Acetate & \multicolumn{2}{|c|}{ Formate } & Urea \\
\hline \multicolumn{2}{|c|}{ Chem. Shift (ppm) } & 183.7 & 178.0 & 174.1 & 172.5 & 165.0 \\
\hline \multicolumn{2}{|c|}{ Effective $T_{1}(\mathrm{~s})$} & 40.7 & 22.8 & 17.6 & 18.1 & 60.1 \\
\hline \multirow{2}{*}{$\begin{array}{l}\text { Absolute } \\
\text { intensity* }\end{array}$} & $\tau=9.1 \mathrm{~s}$ & 0.7998 & 0.6713 & 0.5961 & 0.6045 & 0.8595 \\
\hline & $\tau=9.3 \mathrm{~s}$ & 0.7959 & 0.6654 & 0.5894 & 0.5979 & 0.8566 \\
\hline \multirow{2}{*}{$\begin{array}{l}\text { Relative } \\
\text { intensity }\end{array}$} & $\tau=9.1 \mathrm{~s}$ & 0.2265 & 0.1901 & 0.1688 & 0.1712 & 0.2434 \\
\hline & $\tau=9.3 \mathrm{~s}$ & 0.2271 & 0.1898 & 0.1681 & 0.1706 & 0.2444 \\
\hline \multicolumn{2}{|c|}{$\begin{array}{l}\text { Intensity variation for a transfer } \\
\text { time varying from } 8.2 \text { to } 10.2 \mathrm{~s}\end{array}$} & $0.2 \%$ & $-0.1 \%$ & $-0.4 \%$ & $-0.4 \%$ & $0.4 \%$ \\
\hline
\end{tabular}

*The absolute intensity is calculated by $e^{-\tau / \mathrm{T} 1}$, where $\mathrm{t}$ is the transfer time and T1 the effective T1. 